\title{
Orphan afterglows: the off-axis view of Gamma Ray Bursts
}

\section{G. Ghirlanda*i}

INAF-Osservatorio Astronomico di Brera, via E. Bianchi 46. I-23807 Merate (LC)

E-mail: giancarlo.ghirlanda@brera.inaf.it

GRBs detected in the $\gamma$-ray band are those "seen" within their jets (which have typical opening angles $\theta_{\text {jet }} \sim 2-10$ degrees). When seen off-axis, the high relativistic beaming (due to bulk Lorentz factors $\Gamma>100$ ) prevents the detection of the high energy $\gamma$-ray emission. However, the slow down of the outflow (due to the interaction with the interstellar matter) makes the afterglow potentially observable a few days after the GRB itself. Deep wide field radio surveys have the potential to detect the off-axis population of Gamma Ray Bursts (dubbed Orphan Afterglows $\mathrm{OA}$ ) as relatively slow (days to months timescales) transients (at a flux density level of 10-100 $\mu \mathrm{Jy}$ at $\mathrm{GHz}$ frequencies) with a detection rate of few events per year. The detection rate of OA should increase from the the VLASS $\left(\sim 16 \mathrm{yr}^{-1}\right)$ through the VAST-Wide $\left(\sim 25-45 \mathrm{yr}^{-1}\right)$ to the SKA1 $\left(\sim 220 \mathrm{yr}^{-1}\right.$ ) survey. On the other hand, low frequency (MHz) surveys have less chances to detect these transients. Spectroscopy and multi wavelength follow up will be necessary to distinguish OA from extragalactic transients (SN, TDE and AGN flares) with similar temporal properties and brightness. Detection and study of the OA population in the radio band is of primary importance for the potential support in the identification of the counterparts of gravitational wave events.

EXTRA-RADSUR2015 (*)

20-23 October 2015

Bologna, Italy

(*) This conference has been organized with the support of the Ministry of Foreign Affairs and International Cooperation, Directorate General for the Country Promotion (Bilateral Grant Agreement ZA14GR02 - Mapping the Universe on the Pathway to SKA)

\footnotetext{
* Speaker.
} 


\section{GRB Orphan Afterglows (OA)}

The observational picture of Gamma Ray Bursts (GRBs) has been enriched in the recent years by the discovery of new features of the prompt $\gamma$-ray emission (e.g. by the Fermi satellite) and by the detailed monitoring of the afterglow emission from the X-ray (e.g. Swift) to the optical (ground based telescope networks) starting soon after the burst discovery.

The study of their $\gamma$-ray emission (prompt phase) showed that the isotropic equivalent luminosities are in the range $10^{46-53} \mathrm{erg} / \mathrm{s}$. The study of their early optical/X-ray (afterglow) light curves (Molinari et al. 2007; Ghirlanda et al. 2012) supported the argument (Sari \& Piran 1999) that their outflows are relativistic with typical bulk Lorentz factors reaching $>100-500$ during the production of the $\gamma$-ray emission. Late time observations of the fading afterglow emission showed, in some cases, the signatures of jetted outflow with typical opening angles of few degrees. After all, GRBs are powerful relativistic jetted sources.

The detection rate of GRBs by present flying satellites are in the range $\sim 15-30 \mathrm{yr}^{-1} \mathrm{Sr}^{-1}$. This is an approximate average estimate which depends on the detector type and is mainly limited by the detection threshold of a given instrument and by its field of view. However, since the $\gamma$-ray emission of GRBs is produced within a narrow jet, such a rate corresponds to only those bursts whose jet is pointed to the Earth. Considering the typical opening angle $\theta_{\text {jet }} \sim 2-6^{\circ}$ (e.g. Ghirlanda et al. 2007; Ghirlanda et al. 2012), for each event detected as a GRB, there should be $\sim 1 /\left(1-\cos \theta_{\text {jet }}\right)$ more events (e.g. for $\theta_{\text {jet }} \sim 0.1$ these are $\sim 200$ ) which goes undetected because oriented "off-axis", i.e. whose jet does not intercepts the observer's line of sight.

Despite being so numerous, off-axis GRBs are not completely unobservable. According to the standard model, the afterglow emission is produced by the deceleration of the relativistic outflow by the interstellar medium: the bulk Lorentz factor decreases from a few hundred (during the prompt phase) to sub relativistic values years after the burst. In turn, the beaming of the radiation produced during this phase decreases: there will be a characteristic timescale when even the offaxis observer will see photons because the beaming factor $\propto 1 / \Gamma$ is equal to the viewing angle $\theta_{\text {view }}$, i.e. the angle between the jet axis and the line of sight. Therefore, at relatively late times after the burst, also the off-axis bursts can become visible: they should appear as transients (with typical rise and decay timescales of days-weeks depending on the observing frequency) but without any associated prompt $\gamma$-ray emission which is "lost" forever, i.e. it has been emitted early on and with a beaming factor $1 / \Gamma \ll \theta_{\text {view }}$.

\section{Perspective for detection of $\mathrm{OA}$ in radio surveys}

Searches of OA in wide field surveys in the X-ray (e.g. Grindlay 1999; Greiner et al. 2000) and optical (Rau et al. 2006, 2009; Malacrino et al. 2007) resulted in no clear detection (the few candidates were excluded to be OA based on follow up and spectroscopic studies). In the radio band, the afterglow emission is initially self absorbed: the peak of the flux is observed days after the burst and, overall, the timescale of the flux evolution (rise and decay) can be followed over long timescales. Searches for OA in the radio band (Levinson et al. 2002; Gal-Yam et al. 2006) resulted in no detection. Recent possible detection of OA does not exclude other possible interpretations 
(e.g. an under luminous GRB - Cenko et al. 2013). In general, OA have been searched as on-off transients in wide field surveys.

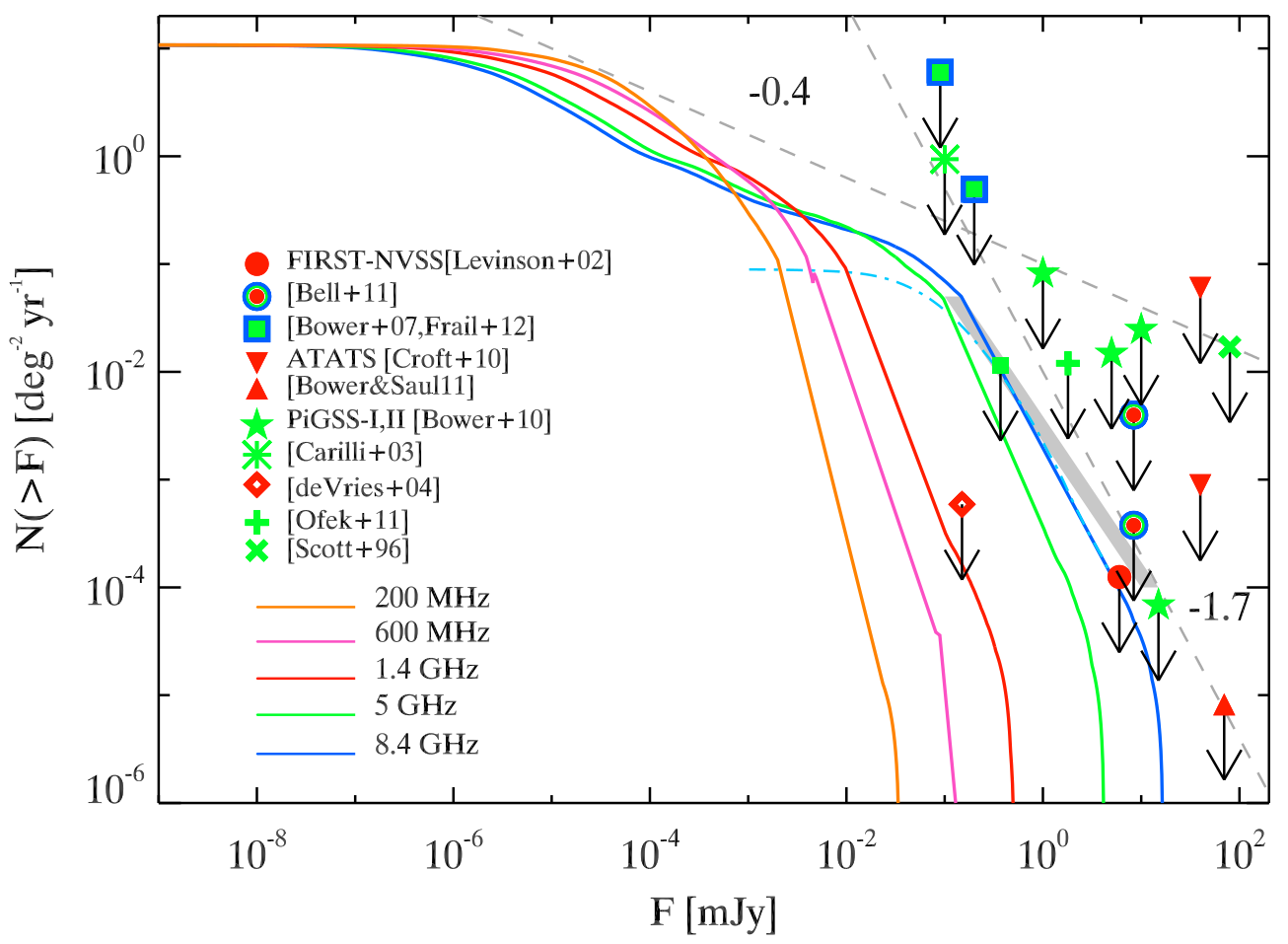

Figure 1: Cumulative flux density distribution of the population of orphan afterglows (OA) from Ghirlanda et al. 2014. Different solid curves correspond to different frequencies (as shown in the legend). Upper limits (at the $3 \sigma$ level) derived from past searched of OA in wide field radio surveys are shown by different symbols (see the legend). Dashed lines show for reference the typical slopes of the high frequency curves above and below the break. The shaded grey line is the flux distribution computed by Frail et al. (2012) for the $8.4 \mathrm{GHz}$ frequency.

Oversimplifying, the lack of OA detections so far could be either due to the shallow flux sensitivity of the surveys conducted in the past or to some physical property of GRBs. The latter could be related to the structure of the jet of GRBs (e.g. Rossi et al. 2008). While a typical conical jet with uniform $\Gamma$ and energy distribution (as a function of the angle from the axis) is assumed for GRB jets, it is also possible, and even more likely for the long GRB population due to the interaction of the jet with the stellar envelope, that the jet has a structure. Present studies of the GRB luminosity function (Pescalli et al. 2015) and GRB energetics (Salafia et al. 2015) seem to favour a steep jet structure, i.e. a conical narrow jet with some energy distributed in gaussian tails. In this case, the number of OA is smaller than in the case of a uniform jet because even off-axis there is some portion of the jet emitting radiation in the observer direction.

The rate of OA can be predicted by modelling the population of GRBs. OA represent the parent population of GRBs: a population synthesis model that reproduces the observed properties of the prompt and afterglow emission of GRBs (Ghirlanda et al. 2013) allows us to extrapolate the 
GRB properties to the off-axis population (Ghirlanda et al. 2014, 2015).

Fig.1 (from Ghirlanda et al. 2014) shows the cumulative distribution of the rate of OA (in units of $\mathrm{yr}^{-1} \mathrm{deg}^{-2}$ ) as a function of the flux density. The latter is computed at the time when the OA is at its maximum brightness. The different curves (color) show different radio frequencies from hundreds of $\mathrm{MHz}$ to tens of $\mathrm{GHz}$. For the highest frequencies, the bright end of the flux density distribution extends to the mJy level despite this corresponds to a very low rate. The curves in Fig.1 allow us to estimate the rate of OA with flux density larger than a given limit. For instance, at 8.4 $\mathrm{GHz}$ we estimate $\sim 2 \times 10^{-3} \mathrm{OA} \mathrm{yr}^{-1} \mathrm{deg}^{-2}$ with flux density brighter than $1 \mathrm{mJy}$. These curves were obtained with realistic assumptions on the distribution of the jet opening angles, luminosity function and redshift distribution of GRBs and are consistent, in the bright flux end, with past estimates (e.g. Frail et al. 2012 - shown by the shaded grey line in Fig.1).

Fig.1 shows that:

- a broken power law distribution with typical slopes -1.7 and -0.4 above and below a characteristic flux (whose value is frequency dependent). This break is due to the convolution of the peaked $\theta_{\text {jet }}$ distribution with the probability density function of the orientation of the jet in the plane of the sky $\left(\propto \sin \theta_{\text {view }}\right)$;

- the predicted curves are all consistent with the upper limits derived from the searches of transients (among which also OA) in wide field radio surveys (symbols corresponding to the legend in Fig.1);

- the relative flux densities represented by the different curves (i.e. with higher frequency curves corresponding to larger flux densities) are due to the fact that the afterglow emission is in the self-absorbed regime in the radio band. This shows that at $\mathrm{MHz}$ frequencies (200 and $600 \mathrm{MHz}$ shown in Fig.1) the OA are dim transients.

The future of wide field deep survey in the radio band is bright. LOw Frequency ARray (LOFAR; van Haarlem et al. 2013), the Murchison Widefield Array (MWA) are going to provide unprecedented sensitive wide field surveys at meter wavelength. At $\mathrm{GHz}$ frequencies upgrades of existing facilities (e.g the Apertif project at the Westerbork Synthesis Radio Telescope and the Karl G. Jansky Very Large Array - Perley et al. 2011) offer new opportunities to detect and study the transient radio sky also in view of the forthcoming MeerKAT and the Australian Square Kilometer Array Pathfinder (ASKAP; Johnston et al. 2008), both precursors of the Square Kilometer Array.

In order to predict the rate of detection of OA by a given survey, in addition to the flux density distributions shown in Fig.1, we also need to know the typical timescale over which the OA will appear and disappear above and below a given survey threshold. This has been computed in Ghirlanda et al. (2014; 2015) and it is shown in Fig.2. For two different frequencies, Fig.2 shows that, for instance at $8.4 \mathrm{GHz}$, a survey with a flux limit of $10^{-2} \mathrm{mJy}$ would detect OA with an average duration of a couple of weeks. Considering a typical survey which can reach this flux limit and that covers a fraction of the sky with a certain cadence we can estimate the rate of OA that it will detect by multiplying the rate (estimated from Fig.1) by the survey coverage (i.e. degrees per day) and by the average duration of the OA above the survey flux limit (see e.g. Ghirlanda et al. 2015). 


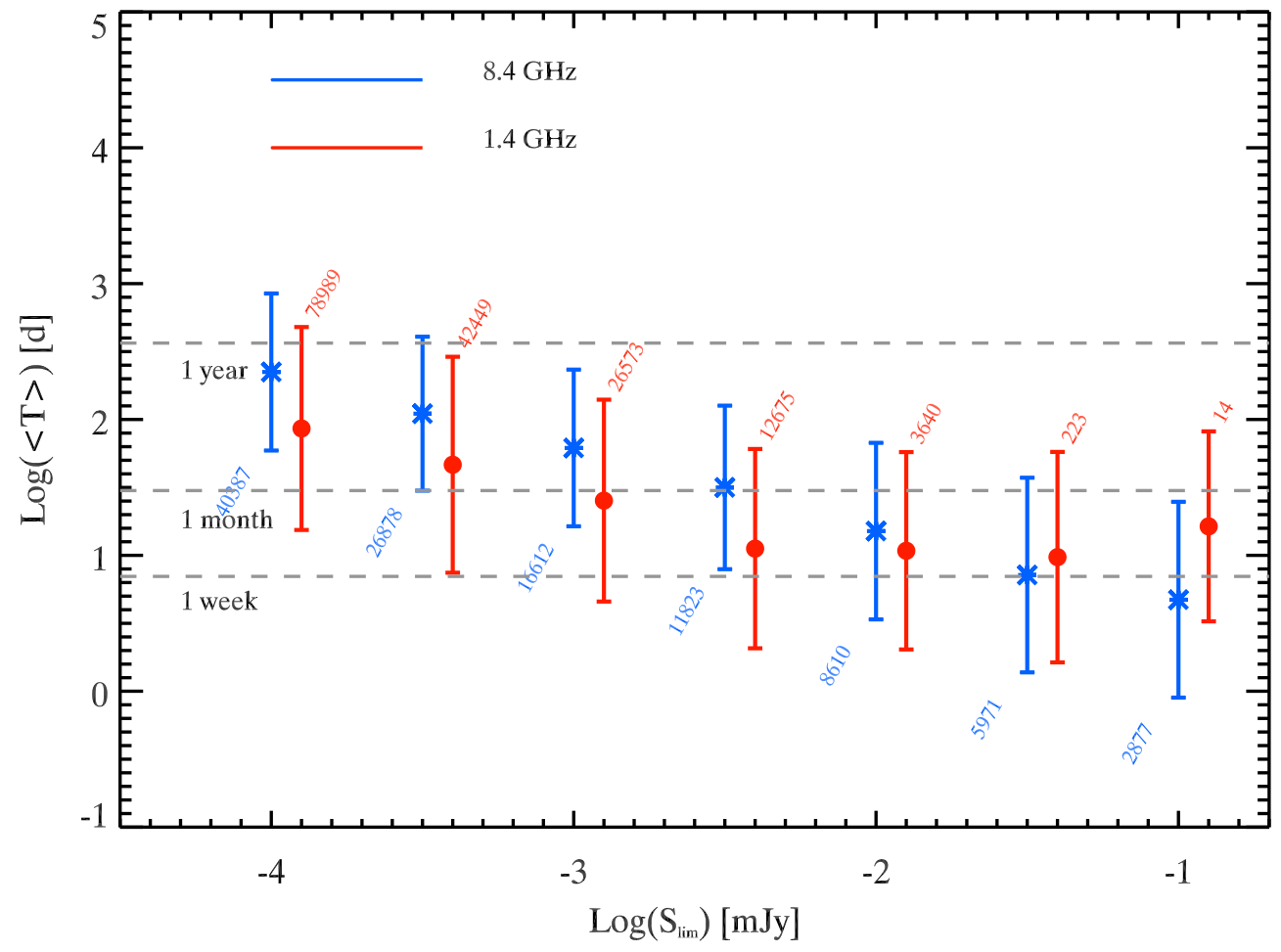

Figure 2: Cumulative distribution of the average duration of the OA as transients for a survey with a threshold represented on the $\mathrm{x}$-axis. Two frequencies are shown. Timescales are in days - from Ghirlanda et al. 2014. Error bars show the width of the distributions of the timescales for each flux. The numbers reported near each symbols represent the OA expected above the corresponding flux limit over the entire sky.

If we consider $150 \mathrm{MHz}$ as the typical frequency of the LOFAR (van Haarlem et al. 2013) and MWA surveys that should cover $\sim 1500 \mathrm{deg}^{2}$ per month reaching a flux limit of $12 \mathrm{mJy}$ (rms), from Fig. 1 and Fig. 2 we estimate that no OA is expected. At $1.4 \mathrm{GHz}$ we can consider the VAST-Wide and the VAST-Deep surveys (both to be performed with the ASKAP - Murphy et al. 2013) which should cover $10^{4} \mathrm{deg}^{2} \mathrm{day}^{-1}$ and $30 \mathrm{deg}^{2} \mathrm{day}^{-1}$ down to 0.5 and $0.05 \mathrm{mJy}$, respectively. These should detect 45 to $26 \mathrm{OA} \mathrm{yr}^{-1}$ (all estimates assume the rms) respectively. In perspective, the SKA-1 survey will cover $10^{4} \mathrm{deg}^{2}$ every 90 days down to $0.009 \mathrm{mJy}$ thus detecting up to $220 \mathrm{OA}$ $\mathrm{yr}^{-1}$ (Burlon et al. 2015). At higher frequencies, already the VLA survey (VLASS-AS - Metzger et al. 2015) at $3 \mathrm{GHz}$ will detect $15 \mathrm{OA} \mathrm{yr}^{-1}$ (covering $10^{4} \mathrm{deg}^{2} \mathrm{yr}^{-1}$ down to $0.1 \mathrm{mJy}$ ).

\section{Discussion and Conclusions}

Orphan afterglows are relatively slow transients, they have timescales between days and several months (up to years). Among extragalactic transients showing similar temporal properties and luminosities there are Blazars, AGN in flaring states and Supernovae. Follow up campaigns in the $\mathrm{X}$-ray and optical (through dedicated facilities and/or observing programs) will secure light curves and spectra of the detected transients. The time evolution during the decay phase typical of GRB 
afterglows (e.g. $\propto t^{-1}$ ) and the spectroscopic study of the featureless afterglow spectrum would help to identify OA (e.g. see Ghirlanda et al. 2015). Contemporaneous detection of the same set of transients in surveys performed at different frequencies (e.g. Gaia, LSST among the most promising for the optical and eROSITA for the X-ray - see Ghirlanda et al. 2015 for specific predictions) will allow us also to verify the afterglow signatures in the time domain. In particular, low redshift transients will be aided by the possible identification of the host galaxy which could provide further hints on the transient nature.

\section{References}

[1] E. Molinari, et al., REM observations of GRB 060418 and GRB 060607A: the onset of the afterglow and the initial fireball Lorentz factor determination, ApJ 469 (2007) L13.

[2] G. Ghirlanda, et al., Gamma-ray bursts in the comoving frame MNRAS 420 (2012) 483.

[3] R. Sari \& T. Piran, The early afterglow A\&A 138 (1999) 537.

[4] G. Ghirlanda, et al., Confirming the $\gamma$-ray burst spectral-energy correlations in the era of multiple time breaks A\&A 466 (2007) 127.

[5] J. Grindlay, Fast X-Ray Transients and Gamma-Ray Bursts: Constraints on Beaming ApJ 510 (1999) 710.

[6] J. Greiner, et al., Search for GRB X-ray afterglows in the ROSAT all-sky survey A\&A 353 (2000) 998.

[7] A. Rau, et al., Constraining the GRB collimation with a survey for orphan afterglows A\&A 449 (2006) 79.

[8] A. Rau, et al., Exploring the Optical Transient Sky with the Palomar Transient Factory PASP 121 (2009) 1334.

[9] F. Malacrino, et al., Constraining the rate of GRB visible afterglows with the CFHTLS very wide survey A\&A 464 (2007) L29.

[10] A. Levinson, et al., Orphan Gamma-Ray Burst Radio Afterglows: Candidates and Constraints on Beaming ApJ 576 (2002) 923.

[11] A. Gal-Yam, et al., Radio and Optical Follow-up Observations of a Uniform Radio Transient Search: Implications for Gamma-Ray Bursts and Supernovae ApJ 639 (2006) 331.

[12] A. Gal-Yam, et al., Radio and Optical Follow-up Observations of a Uniform Radio Transient Search: Implications for Gamma-Ray Bursts and Supernovae ApJ 639 (2006) 331.

[13] B. Cenko, et al., Discovery of a Cosmological, Relativistic Outburst via its Rapidly Fading Optical Emission ApJ 796 (2013) 130.

[14] G. Ghirlanda, et al., GRB Orphan Afterglows in Present and Future Radio Transient Surveys PASA 31 (2014) 22.

[15] D. Frail, et al., A Revised View of the Transient Radio Sky ApJ 747 (2012) 70.

[16] E. Rossi, et al., 'Orphan' afterglows in the Universal structured jet model for $\gamma$-ray bursts MNRAS 390 (2008) 675.

[17] A. Pescalli, et al., Luminosity function and jet structure of Gamma-Ray Burst MNRAS 447 (2015) 1911. 
[18] O. S. Salafia, et al., Structure of Gamma-Ray Burst jets: intrinsic versus apparent properties MNRAS 450 (2015) 3549.

[19] O. S. Salafia, et al., Structure of Gamma-Ray Burst jets: intrinsic versus apparent properties MNRAS 450 (2015) 3549.

[20] G. Ghirlanda, et al., Unveiling the population of orphan $\gamma$-ray bursts A\&A 578 (2015) A71.

[21] M. P. van Haarlem, et al., LOFAR: The LOw-Frequency ARray A\&A 556 (2013) 2.

[22] R. A. Perley, et al., The Expanded Very Large Array: A New Telescope for New Science ApJ 739 (2011) L1.

[23] T. Murphy, et al., VAST: An ASKAP Survey for Variables and Slow Transients PASA 30 (2013) 6.

[24] D. Burlon, et al., The SKA View of Gamma-Ray Bursts In the Proceedings of Advancing Astrophysics with the Square Kilometre Array AASKA1 4.052 (2015).

[25] B. Metzger, et al., Extragalactic Synchrotron Transients in the Era of Wide-field Radio Surveys. I. Detection Rates and Light Curve Characteristics ApJ 806 (2015) 224. 